\title{
Pasión por el Trabajo: Un Estudio Instrumental con Trabajadores Argentinos
}

\section{Work Passion: An Instrumental Study with Argentinean Workers}

\author{
Solana Salessi \\ Consejo Nacional de Investigaciones Científicas y Técnicas y Centro de Investigaciones y \\ Transferencia de la Universidad Nacional de Rafaela \\ Alicia Omar \\ Consejo Nacional de Investigaciones Científicas y Técnicas y Universidad Nacional de Rosario
}

\begin{abstract}
Se presentan los resultados de la validación de la escala de Pasión por el Trabajo de Vallerand et al. (2003). Para determinar la validez de constructo y las propiedades psicométricas de la escala se trabajó con una muestra multiocupacional por conveniencia de 452 trabajadores argentinos: $56 \%$ mujeres; promedio de edad $=35,56$ años $(D E=10,84)$; promedio de antigüedad en el trabajo $=8,82$ años $(D E=9,10)$. Análisis factoriales indicaron una estructura de 2 factores latentes correlacionados integrados por 14 ítems como indicadores observables $\left(\mathrm{S}-\mathrm{B} \chi^{2} / g l=\right.$ $1,85, \mathrm{GFI}=0,90, \mathrm{CFI}=0,94, \mathrm{RMSEA}=0,04)$, con adecuada confiabilidad según el coeficiente alfa ordinal y el coeficiente de confiabilidad compuesta (pasión armoniosa: $\alpha=0,79, \mathrm{CR}=0,80$; pasión obsesiva: $\alpha=0,80, \mathrm{CR}=0,81$ ). Se obtuvo evidencia de validez concurrente a partir de las correlaciones positivas calculadas mediante el coeficiente rho de Sperman entre cada una de las escalas de pasión por el trabajo, flow, implicación en el trabajo y satisfacción laboral. Se comprobó la validez predictiva e incremental de la pasión más allá del flow y de la implicación en el trabajo en la explicación de la satisfacción laboral según el valor del $\mathrm{R}^{2}$. Se demostró la invarianza configural y métrica de la escala entre varones y mujeres según la prueba de diferencia de $\chi^{2}$. Los resultados obtenidos dan cuenta de la confiabilidad y la validez de este instrumento para medir la pasión por el trabajo en un grupo de trabajadores argentinos.
\end{abstract}

Palabras clave: pasión por el trabajo, escala, validez factorial, consistencia interna, análisis psicométrico

\begin{abstract}
This article presents the results of the validation of the Work Passion Scale developed by Vallerand et al. (2003). To determine the construct validity and the psychometric properties of the scale, a convenience multi-occupational sample of 452 Argentinean workers ( $56 \%$ women, average age $=35.56$ years $[S D=10.84$ ], average seniority $=8.82$ years $[S D=9.10]$ ) was used. Factor analyses revealed a structure composed of 2 correlated latent factors, comprising 14 items as observable indicators $(\mathrm{S}-\mathrm{BX} / g l=1.85, \mathrm{GFI}=0.90$, CFI $=0.94$, RMSEA $=0.04)$, with adequate reliability according to the ordinal alpha coefficient and the composite reliability coefficient (harmonious passion: $\alpha=0.79, \mathrm{CR}=0.80$; obsessive passion: $\alpha=0.80, \mathrm{CR}=0.81$ ). Evidence of concurrent validity was obtained from direct correlations, calculated using Spearman's rho, between the scales of passion for work, flow, job involvement, and job satisfaction. According to the $R^{2}$ coefficient, the predictive and incremental validity of passion as an explanation of job satisfaction surpassed the impact of flow and job involvement. The configural and measurement invariance of the scale across gender was demonstrated using the $X^{2}$ difference test. The results obtained prove the reliability and validity of this instrument to measure passion for work in a group of Argentinean workers.
\end{abstract}

Keywords: passion for work, factor validity, psychometric analysis

La pasión constituye una noción omnipresente en el discurso popular. Durante siglos, filósofos, dramaturgos y escritores se han interesado por explorar su rol en la vida de las personas. Sin embargo, el

Solana Salessi, Consejo Nacional de Investigaciones Científicas y Técnicas. Centro de Investigaciones y Transferencia de la Universidad Nacional de Rafaela, Rafaela, Argentina. Departamento de Educación, Cultura y Conocimiento. Facultad de Derecho y Ciencias Sociales de la Pontificia Universidad Católica Argentina, Rosario, Argentina.

Alicia Omar, Consejo Nacional de Investigaciones Científicas y Técnicas. Instituto de Investigaciones de la Facultad de Humanidades y Artes, Universidad Nacional de Rosario, Rosario, Argentina.

La correspondencia relativa a este artículo debe ser dirigida a Solana Salessi, Pontificia Universidad Católica Argentina, Rosario, Argentina; Facultad de Derecho y Ciencias Sociales. Av. Pellegrini 3314, S2002QEO Rosario, Santa Fe - Tel: 340415500180 solanasalessi@uca.edu.ar 
estudio científico de este constructo es reciente. Salvo algunas excepciones, las primeras publicaciones psicológicas sobre pasión se remontan a principios de este siglo (Vallerand et al., 2003; Vallerand \& Houlfort, 2003), siendo Vallerand uno de los principales investigadores en abocarse a su estudio científico.

La pasión se define como una fuerte inclinación hacia una actividad que genera placer (Vallerand, 2015). Para que una actividad constituya una pasión es imprescindible que sea relevante para el sujeto y que, además, se le dedique tiempo y esfuerzo en su realización de manera regular y sostenida. A la luz de estos criterios, parece plausible que el trabajo pueda representar una pasión, habida cuenta de que, además de ser la actividad productiva por excelencia y el principal organizador de la cotidianeidad, constituye un ámbito esencial de realización y desarrollo de la personalidad. En este sentido, la pasión por el trabajo se define como un estado de deseo persistente, basado en valoraciones cognitivas y afectivas del propio trabajo (Perrewé, Hochwarter, Ferris, McAllister \& Harris, 2014). Se trata de un estado positivo y significativo de bienestar, derivado de las evaluaciones que el sujeto efectúa de su situación laboral y que resulta en un trabajo consistente y constructivo (Zigarmi, Nimon, Houson, Witt \& Diehl, 2009).

Siguiendo los postulados del modelo dualista (Vallerand, 2015; Vallerand et al., 2003; Vallerand \& Houlfort, 2003), el mecanismo explicativo subyacente a la pasión es la internalización de la actividad (en este caso, el trabajo) en el self. No obstante, el modo como se lleva a cabo este proceso tiñe de connotaciones particulares a la pasión, haciendo posible distinguir dos variantes: la pasión armoniosa y la pasión obsesiva. Mientras que en la primera la internalización de la actividad en la identidad sucede de manera autónoma, en la segunda ocurre de manera controlada y compulsiva. Por este motivo, la pasión armoniosa tiende a ser caracterizada como activa y adaptativa, en tanto que la pasión obsesiva, como pasiva y esclavizante.

En la pasión armoniosa el individuo no experimenta un impulso incontrolable a realizar la actividad apasionante. La actividad ocupa un espacio significativo, pero no abrumador en su identidad, encontrándose en equilibrio con otros aspectos de su vida. La persona puede comprometerse en ella con la suficiente apertura para experimentar el mundo en forma no defensiva (Vallerand, 2015). La pasión armoniosa por el trabajo lleva a las personas a invertir esfuerzos de manera sostenida, permitiendo adquirir nuevas habilidades, desarrollar las propias fortalezas naturales y favorecer sentimientos de competencia y eficacia personal. Sumado a eso, la participación en la actividad que se ama con la flexibilidad que esta pasión supone proporciona sentimientos de autonomía y emociones positivas (Curran, Hill, Appleton, Vallerand \& Standage, 2015). En contraposición, aun valorándola como un aspecto importante y significativo en su vida, el individuo obsesivamente apasionado se siente obligado a realizar la actividad, incluso, en momentos en que no debería. La decisión de participar en ella no responde al libre albedrío, sino a la necesidad imperiosa de hacer frente a las presiones que lo subyugan. A diferencia de la anterior, la pasión obsesiva puede acaparar un espacio desproporcionado en la identidad y ocasionar conflictos con otras esferas vitales. En efecto, las personas obsesivamente apasionadas se aferran a su pasión, experimentando menor interés por otras actividades (Vallerand \& Houlfort, 2003).

Si bien en ambos tipos de pasión subsisten necesidades psicológicas básicas que la actividad apasionante debe satisfacer (Forest, Mageau, Sarrazin \& Morin, 2011), en la pasión obsesiva las presiones que compelen a participar en ella se tornan imperiosas e incontrolables. En especial, las personas que cultivan este tipo de pasión se refugian en el trabajo, buscando proteger e incrementar su propia estima personal. De aquí que esta presente notables fluctuaciones al son de las contingencias de la actividad apasionante (Mageau, Carpentier \& Vallerand, 2011). Mientras puedan cumplir los objetivos propuestos y desempeñarse satisfactoriamente, los empleados obsesivamente apasionados experimentarán una mejora significativa en su autoestima. De lo contrario, esta se verá perjudicada, motivando un mayor ensimismamiento en el trabajo. En efecto, la evidencia empírica indica que la pasión obsesiva se relaciona a motivaciones de escape o huida. De aquí que, en estos casos, la actividad apasionante se imponga como un refugio potencial frente a la falta de satisfacción en otras áreas de su vida (Lalande et al., 2017).

La pasión por el trabajo se asocia a un amplio abanico de conductas concomitantes, que incluyen desde la mera perseverancia en la realización de las tareas hasta la involucración del individuo en comportamientos discrecionales que exceden los prescriptos para su rol. No obstante, también puede relacionarse a resultados negativos, tanto para el propio empleado como para las organizaciones. En este sentido, las personas obsesivamente apasionadas son proclives a experiencias de temor e inseguridad. Su frágil autoestima las torna vulnerables a los cambios y a la incertidumbre, reforzando su rigidez y fomentando actitudes suspicaces. Aun mostrando una elevada constancia, sus niveles de concentración y fluidez tienden a verse perjudicados. Merced a la disminución de su eficacia y a los magros resultados 
obtenidos en su desempeño, los sentimientos depresivos y las intenciones de abandonar la organización están a la orden del día entre estos empleados (Houlfort, Philippe, Vallerand \& Ménard, 2014). Las personas armoniosamente apasionadas, en cambio, tienden a protagonizar en mayor medida experiencias de afecto positivo. Al tratarse de una internalización libre y autónoma, la pasión armoniosa permite que la persona participe en la actividad de una manera más flexible y adaptativa. Por ello, esta forma de pasión actúa como una fuerza motivadora que impulsa a involucrarse en la actividad con entusiasmo, favoreciendo la concentración y la implicación positiva en la tarea (Dubreuil, Forest \& Courcy, 2014).

\section{La Pasión por el Trabajo en el Entramado de la Psicología Organizacional Positiva}

El constructo pasión por el trabajo ha sido propuesto por Vallerand y Verner-Filion (2013) como candidato legítimo para incorporarse a la red nomológica de la psicología positiva. Sin embargo, aun cuando su validez de constructo ha sido demostrada por numerosos estudios (Marsh et al., 2013; OrgambídezRamos, Borrego-Alés \& Gonçalves, 2014; Vallerand et al., 2003; Zito \& Colombo, 2017), su semejanza con otros conceptos es objeto de controversias. Entre los constructos afines, engagement, adicción al trabajo y compromiso organizacional merecen especial atención.

El engagement describe un estado mental positivo de realización relacionado con el trabajo, siendo sus principales notas distintivas el vigor, la dedicación y la absorción en la tarea (Bakker \& Albrecht, 2018). Si bien engagement y pasión comparten su naturaleza motivacional, presentan dos diferencias esenciales. En primer lugar, la pasión presupone como requisito ineludible que el trabajo sea parte fundamental de la identidad y del autoconcepto del individuo (Vallerand, 2015; Vallerand et al., 2003), condición que no se observa entre los trabajadores engaged. En segundo lugar, el engagement constituye un estado transitorio que oscila diariamente al ritmo de las experiencias laborales (Engeser \& Baumann, 2016), en tanto que la pasión se encuentra exenta del influjo de los pormenores cotidianos (Vallerand \& Houlfort, 2003), de modo que, mientras el engagement refleja más la intensidad de las experiencias laborales, la pasión alude a la calidad de la relación con el trabajo (Salessi, Omar \& Vaamonde, 2017).

La adicción (del inglés workaholic; Andreassen, 2014) se caracteriza por trabajar excesivamente duro y por experimentar un irresistible impulso a hacerlo. Un adicto al trabajo es una persona que: (a) invierte considerable cantidad de tiempo en actividades relacionadas con el trabajo con consecuencias negativas para su desarrollo social y familiar; (b) continúa concentrándose en el trabajo, aun en sus momentos de ocio, y (c) trabaja más allá de las expectativas, necesidades o demandas de la organización. Si bien la compulsión por el trabajo es el denominador común entre pasión obsesiva y adicción, esta última es incompatible con experiencias de placer y disfrute (Taris, van Beek \& Schaufeli, 2014). Contrariamente, en la pasión el placer constituye un aspecto central, incluso entre los obsesivamente apasionados (Salessi \& Omar, 2018a; Orgambídez-Ramos et al., 2014; Zito \& Colombo, 2017).

Finalmente, el compromiso organizacional se refiere a los lazos que por deseo, necesidad u obligación mantienen al sujeto en la organización y que tiene repercusiones en su decisión de mantener o no su condición de miembro de esta (Meyer, 2016). La pasión, en cambio, atañe al vínculo de la persona con su trabajo, independientemente de la organización en la que lo ejerza. En efecto, se ha demostrado (Lajom, Amarnani, Restubog, Bordia \& Tang, 2018) que el compromiso tiende a ser una consecuencia de la pasión, antes que un determinante de esta.

Además de estos constructos, otros conceptos que integran el entramado nomológico de la psicología positiva ameritan un examen minucioso de sus semejanzas y diferencias con la pasión. Entre estos, el flow relacionado con el trabajo (Bakker, 2008) y la implicación (del inglés job involvement; Kanungo, 1982) revisten especial interés. El flow se refiere a una experiencia óptima a corto plazo, caracterizada por la inmersión absoluta en el trabajo, las emociones positivas y la motivación intrínseca frente a la tarea (Bakker, 2008). La implicación en el trabajo, en tanto, hace referencia al grado en que una persona está cognitivamente involucrada con su trabajo actual (Kanungo, 1982). Se trata de una creencia descriptiva determinada situacionalmente, ligada a la percepción del potencial de un trabajo particular para satisfacer las necesidades más salientes del individuo (Salessi \& Omar, 2018b).

Si bien tanto el flow como la pasión presuponen experiencias de placer, el primero constituye un fenómeno temporal circunscripto al tiempo que insuma la tarea (Bakker, 2008; Engeser \& Baumann, 2016). En contraste, la pasión es un estado psicológico de deseo por el trabajo que persiste en el tiempo y trasciende las actividades particulares (Vallerand et al., 2003; Vallerand \& Houlfort, 2003). Del mismo 
modo, aun cuando en la implicación el trabajo ocupa un lugar prominente en la vida de la persona, no constituye un componente central de su identidad, situación que sí se observa en la pasión, donde la internalización del trabajo en el self es su nota distintiva (Vallerand, 2015).

\section{La Medición de la Pasión por el Trabajo}

Como corolario de sus esfuerzos por desarrollar una sólida base conceptual para el novedoso constructo, Vallerand et al. (2003) se abocaron a la construcción y validación de un instrumento de medida, enmarcado en los postulados del modelo dualista. En este sentido, la Escala de Pasión (Vallerand et al., 2003) es, hasta la fecha, la única herramienta disponible para la evaluación de este constructo en el ámbito laboral. Se trata de una escala tipo Likert presentada bajo el formato acuerdo-desacuerdo, constituida por 14 ítems distribuidos equitativamente en dos subescalas rotuladas como Pasión armoniosa y Pasión Obsesiva. La pasión armoniosa fue operacionalizada mediante indicadores que resaltan la adaptación y el equilibrio en relación a otras áreas. La pasión obsesiva, en tanto, fue operacionalizada por ítems que enfatizan la falta de control en relación a la actividad apasionante y la dependencia.

La validación inicial (Vallerand et al., 2003) fue realizada con base en los datos proporcionados por una muestra de 539 estudiantes canadienses. Desde entonces, el instrumento ha sido adaptado y validado en diversos países, tales como España (Orgambídez-Ramos et al., 2014; Serrano-Fernández, Boada-Grau, GilRipoll \& Vigil-Colet, 2017), Italia (Zito \& Colombo, 2017), Holanda (van der Knaap \& Steensma, 2015) y Portugal (Martins, Araújo \& Almeida, 2014); obteniéndose evidencias favorables de su validez de estructura interna, así como, de su confiabilidad. No obstante, a la fecha no se dispone de una versión válida y confiable para Argentina. En un intento por llenar este vacío empírico-instrumental, el presente trabajo se propuso: (a) adaptar y validar la Escala de Pasión de Vallerand et al. (2003) para su empleo en el ámbito laboral argentino y (b) analizar las propiedades psicométricas del instrumento.

\section{Diseño del Estudio}

El presente estudio se inscribe en la categoría de investigaciones instrumentales (Ato, López \& Benavente, 2013). El trabajo se estructura en base a dos estudios complementarios, rotulados como Estudio Piloto y Estudio Principal. La ejecución de ambos estudios se llevó a cabo de acuerdo con los lineamientos éticos establecidos por la Asociación Americana de Psicología y las recomendaciones del Consejo Nacional de Investigaciones Científicas y Técnicas de Argentina para las investigaciones en las ciencias sociales y humanas (Resolución 2827/06).

\section{Estudio Piloto}

El objetivo de este estudio fue traducir y adaptar la Escala de Pasión por el Trabajo de Vallerand et al. (2003) para la población argentina. Con tal propósito, se analizó la validez de contenido, la equivalencia semántica y la equivalencia operacional entre el instrumento foráneo y su versión adaptada (Muñiz, Elosua \& Hambleton, 2013).

\section{Método}

Estrategia de análisis. En primer lugar, se efectuaron tareas de revisión bibliográfica y consultas con expertos para establecer el significado del constructo, tanto en la cultura donde fue desarrollado el instrumento como en la cultura objetivo. Como parte de este proceso, los reactivos de la escala también fueron sometidos a revisión crítica, con el fin de establecer la equivalencia de ítems. En este sentido, tres especialistas, dos en psicología del trabajo y las organizaciones y uno en psicometría, recibieron un cuadernillo conteniendo definiciones conceptuales y operacionales de los constructos, así como la totalidad de los ítems que integraban la escala. Se solicitó a los expertos que clasificaran cada ítem en la dimensión correspondiente. Sobre la base de tales evaluaciones, se efectuó un cálculo de consistencia interna a través del estadístico Kappa.

Para establecer la equivalencia semántica entre la versión traducida y la versión original de la escala, se siguió un procedimiento iterativo de traducción y retraducción. En primer lugar, dos traductores profesionales tradujeron los ítems desde su idioma original al idioma de la población objetivo. Seguidamente, otros dos profesionales bilingües retradujeron los ítems desde el español al inglés. 
Finalmente, dos nuevos traductores científicos compararon las dos formas del instrumento "a ciegas", con el propósito de identificar el grado de concordancia entre el ítem original y el ítem traducido. El grado de equivalencia fue determinada en función de dos categorías de análisis: el significado referencial, vinculado con la concordancia en términos de traducción literal entre el ítem original y el ítem traducido, y el significado general, correspondiente a la articulación de ideas entre el ítem original y su retraducción. El primero fue evaluado sobre una escala visual analógica, en la que la equivalencia entre pares fue juzgada de 0 a 100\%. El segundo fue evaluado por dos traductores, en función de cuatro niveles de equivalencia: (a) inalterado, (b) poco alterado, (c) bastante alterado y (d) completamente alterado.

También se estableció la equivalencia operacional, relativa a la posibilidad de mantener estables las características operativas durante la aplicación del instrumento (por ejemplo, la utilización del mismo formato de respuesta, el uso de instrucciones similares a las originales y la aplicación del mismo modo de administración).

Participantes. Se integró una muestra por conveniencia de 62 estudiantes de un postgrado en administración de empresas de la Universidad Nacional de Rosario, quienes además trabajaban en diversas organizaciones. El $71 \%$ de la muestra se desempeñaba en el ámbito privado y el 47,5\% desarrollaba sus actividades en el sector industrial. El $52 \%$ de la muestra estaba conformada por varones. La edad promedio de los participantes era de 33,81 años $(D E=5,64)$, en tanto que la antigüedad laboral media fue de 4,74 años $(D E=2,24)$.

Procedimiento. La recolección de datos tuvo lugar en las instalaciones de la universidad, en los días y horarios habituales de clases. Se explicitó la naturaleza y objetivos del estudio, solicitando la colaboración voluntaria de los alumnos. Quienes aceptaron colaborar recibieron instrucciones precisas con miras a completar de modo individual la versión traducida y adaptada de la Escala de Pasión por el Trabajo (Vallerand et al., 2003). Una vez concluida la administración, se habilitó un espacio para que los participantes pudieran opinar del instrumento, principalmente en lo referente a posibles ambigüedades de sentido, claridad de la redacción y tiempo requerido para completarla. Tales observaciones fueron capitalizadas para perfeccionar el instrumento, facilitando los ajustes semánticos y sintácticos necesarios.

\section{Resultados}

Validez de contenido. El análisis de la literatura especializada (Vallerand, 2015) permitió concluir que el constructo pasión por el trabajo es equivalente en ambas culturas. La revisión del contenido de cada ítem por parte de los tres profesionales indicó que los mismos cubrían los dos dominios de pasión postulados por el modelo dualista (Vallerand et al., 2003; Vallerand \& Houlfort, 2003).

Respecto a la adaptación lingüística, los expertos sugirieron leves modificaciones, de modo que la terminología empleada en la redacción reflejara el vocabulario habitual de la población argentina. Así, por ejemplo, la expresión descubrir perteneciente a un ítem de la subescala de pasión armoniosa ("the new things that I discover in this job allow me to appreciate it even more") fue sustituida por el término aprender ("las cosas nuevas que aprendo con este trabajo hacen que lo valore todavía más"). Del mismo modo, la expresión memorable presente en la misma subescala ("this job allows me to live memorable experiences") fue reemplazada por el vocablo inolvidable ("este trabajo me permite vivir experiencias inolvidables").

Ambos profesionales indicaron que se había mantenido inalterada la concordancia entre cada ítem original y el traducido al español, por lo que concluyeron que la versión argentina de la Escala de Pasión por el Trabajo presentaba una adecuada equivalencia semántica. En lo que hace a la equivalencia operacional, los participantes señalaron que las instrucciones para realizar la tarea estaban claramente redactadas, que no tuvieron inconvenientes para comprender el contenido de los ítems y que la escala tipo Likert utilizada para responder no generaba dificultades.

Consistencia interna. Los índices de concordancia interjueces calculados a partir de la clasificación de los ítems fueron satisfactorios, oscilando entre 0,89 y $0,92(p=0,001)$.

\section{Estudio Principal}

El objetivo de este estudio fue analizar la estructura factorial y determinar las propiedades psicométricas de la versión traducida y adaptada de la Escala de Pasión por el Trabajo (Vallerand et al., 
2003). Con tal propósito, se efectuaron análisis factoriales de carácter exploratorio y confirmatorio y se examinó la confiabilidad, validez e invariancia métrica y configural del instrumento.

\section{Método}

Participantes. Se integró una muestra inicial no probabilística (muestreo por conveniencia) de 487 participantes, trabajadores de distintas empresas públicas y privadas del conglomerado Rosario y Gran Rosario, provincia de Santa Fe, Argentina. Treinta y cinco casos fueron descartados. Los motivos que justificaron su exclusión fueron que ocho de ellos no estaban físicamente completos, faltándole páginas al cuadernillo, siete mostraron estereotipia en la respuesta a los diversos ítems y los restantes 20 no presentaban información en todas las variables. En algunos casos se había omitido firmar el consentimiento informado, en otros faltaba la información sociodemográfica del participante y en otros no se había respondido más de la mitad de los ítems. La muestra definitiva quedó conformada, entonces, por 452 jóvenes y adultos (56\% mujeres). El promedio de edad fue de 35,56 años $(D E=10,84)$, mientras que el de antigüedad laboral fue de 8,82 años $(D E=9,10)$. El $52 \%$ de la muestra tenía estudios superiores (terciarios y/o universitarios). El 61,52\% trabajaba en el sector privado. La distribución por rubros de actividad de los participantes fue la siguiente: $36 \%$ comercio y servicios, $28 \%$ industria, $20 \%$ salud, $16 \%$ educación.

\section{Instrumentos.}

Escala de pasión por el trabajo. Se aplicó la versión autoadministrable (traducida al español y puesta a punto durante el Estudio Piloto) de la escala homónima de Vallerand et al. (2003). El instrumento se encuentra integrado por 14 ítems con formato tipo Likert de 5 puntos (variando desde $1=$ Totalmente en desacuerdo a $5=$ Totalmente de acuerdo), distribuidos proporcionalmente entre las subescalas de Pasión armoniosa (ejemplo: "las cosas que aprendo en este trabajo hacen que lo valore más todavía") y Pasión Obsesiva (ejemplo: "el impulso es tan fuerte que no puedo dejar de hacer este trabajo"). En su versión original (Vallerand et al., 2003), el instrumento demostró adecuados índices de consistencia interna de acuerdo a los valores del coeficiente alfa de Cronbach (pasión armoniosa: $\alpha=0,79$; pasión obsesiva: $\alpha=0,89$ ).

Escala de satisfacción laboral genérica. Se empleó la adaptación argentina (Salessi \& Omar, 2016) de la escala homónima desarrollada por Madonald y MacIntyre (1997). El instrumento evalúa la satisfacción general del empleado con su trabajo a partir de siete ítems (ejemplo: "en mi trabajo puedo aplicar todas mis capacidades y habilidades"), valorados sobre una escala tipo Likert de 5 puntos $(1=$ Totalmente en desacuerdo; $5=$ Totalmente de acuerdo). De acuerdo a las evidencias de validez $\mathrm{y}$ confiabilidad informadas por Salessi y Omar (2016), la estructura de la escala se ajusta apropiadamente a un modelo uni-factorial integrado por 7 variables observables $(\mathrm{SBX} 2 / \mathrm{gl}=1,12$; $\mathrm{GFI}=0,93$; $\mathrm{CFI}=0,94$; RMSEA= 0,03), con adecuada confiabilidad según el coeficiente alfa de Cronbach $(\alpha=0,87)$.

Escala de implicación en el trabajo. Se empleó la adaptación argentina (Salessi \& Omar 2018b) de la escala desarrollada por Kanungo (1982). El instrumento mide el grado en que una persona está cognitivamente comprometida e involucrada con su trabajo. Se encuentra integrado por 10 ítems (ejemplo: "estoy personalmente comprometido con mi trabajo") valorados sobre una escala tipo Likert de 5 puntos $(1=$ Totalmente en desacuerdo; $5=$ Totalmente de acuerdo). De acuerdo a las evidencias de validez $\mathrm{y}$ confiabilidad informadas por Salessi y Omar (2018b), la estructura de la escala se ajusta apropiadamente a un modelo uni-factorial con 9 variables observables $\left(\mathrm{SBX}_{2}=2,14, \mathrm{GFI}=0,91, \mathrm{CFI}=0,92, \mathrm{RMSEA}=0,02\right)$, con adecuada confiabilidad según el coeficiente alfa de Cronbach $(\alpha=0,89)$.

Escala de flow relacionado con el trabajo. Se aplicó la adaptación argentina (Salessi \& Omar, 2018c) de la escala elaborada por Bakker (2008). El instrumento evalúa la percepción del trabajo como una experiencia caracterizada por la absorción en la tarea, el disfrute y la motivación intrínseca. La escala está integrada por 10 ítems distribuidos en tres subescalas: Absorción (4 ítems; ejemplo: "cuando estoy trabajando no pienso en otra cosa"), Disfrute (3 ítems; ejemplo: "cuando estoy trabajando me siento feliz") y Motivación Intrínseca (3 ítems; ejemplo: "mi trabajo me motiva por sí mismo y no por las recompensas que recibo por él"). Los mismos son valorados sobre una escala tipo Likert de 5 puntos $(1=$ Totalmente en desacuerdo; 5 = Totalmente de acuerdo). De acuerdo a las evidencias de validez y confiabilidad informadas por Salessi y Omar (2018c), la estructura de la escala se ajusta apropiadamente a un modelo tri-factorial con 10 variables observables $(\mathrm{SBX} 2=2,14, \mathrm{GFI}=0,91, \mathrm{CFI}=0,92, \mathrm{RMSEA}=0,02)$, con adecuada 
confiabilidad según el coeficiente alfa de Cronbach obtenido para la escala total $(\alpha=0,89)$, y para cada dimensión (absorción: $\alpha=0,80$; disfrute: $\alpha=0,81$; motivación intrínseca: $\alpha=0,78$ ).

Procedimiento. La recolección de los datos se llevó a cabo en el seno de las organizaciones que aceptaron participar de la investigación, en días y horarios pautados con las autoridades y en los lugares físicos que las mismas dispusieron a tal efecto. La participación de los trabajadores fue voluntaria, anónima y confidencial. No se brindaron incentivos de ningún tipo. La administración de las técnicas estuvo a cargo de personal entrenado. En todos los casos, los participantes completaron de manera individual un cuadernillo conteniendo en su primera hoja el objetivo del estudio y las instrucciones para responder, en la segunda hoja el formulario de consentimiento informado para que fuese firmado y en las restantes páginas los ítems correspondientes a los instrumentos, ordenados aleatoriamente.

Análisis de Datos. En primer lugar, se llevó a cabo una serie de análisis preliminares con miras a evaluar la calidad y confiabilidad de los datos. En este sentido, se analizó la presencia de valores perdidos y puntuaciones extremas. Se identificaron los casos atípicos, mediante el cálculo de puntuaciones $Z$ y las distancias de Mahalanobis al cuadrado $\left(D^{2}\right)$ para cada variable. Se consideraron atípicas univariadas aquellas observaciones alejadas más de 3,5 DE de la media y atípicas multivariadas aquellas que presentasen $D^{2}$ con probabilidad igual o inferior a 0,001 (Hair Jr., Black, Babin, Anderson \& Tatham, 2010).

Se analizó la distribución de las variables mediante el cálculo de estadísticos descriptivos (medias y desviaciones estándar), los coeficientes de asimetría y curtosis y el coeficiente de homogeneidad corregido para cada uno de los ítems de la Escala Pasión por el Trabajo. Valores de curtosis y asimetría inferiores a 1,60 y correlaciones directas superiores a 0,30 se consideraron adecuados (Tabachnick \& Fidell, 2013). Se analizó la adecuación del modelo de medida heptafactorial, compuesto por siete factores latentes correlacionados (cada uno correspondiente a las escalas y subescalas de pasión obsesiva, pasión armoniosa, satisfacción laboral, implicación en el trabajo, absorción, disfrute y motivación intrínseca), con sus respectivos ítems como indicadores observables y los correspondientes errores, mediante un análisis factorial confirmatorio (Anderson \& Gerbing, 1982). Finalmente, se ejecutó la prueba de factor único de Harman para evaluar la presencia de posibles sesgos debidos al método común (Podsakoff, MacKenzie \& Podsakoff, 2012) y se obtuvo el ajuste para un modelo de medida unifactorial.

La validez de constructo se determinó con base a análisis factoriales (Pérez-Gil, Chacón Moscoso \& Moreno Rodríguez, 2000). Atendiendo a la recomendación de contar con un mínimo de 200 observaciones para asegurar que la solución factorial fuera estable y generalizable (Lloret-Segura, Ferreres-Traver, Hernández-Baeza \& Tomás-Marco, 2014), se seleccionaron en forma aleatoria simple 226 casos de los 452 presentes en la matriz de datos. Sobre estos se ejecutó un análisis factorial exploratorio (AFE), previa obtención de los índices de adecuación muestral (pruebas de Kaiser-Meyer-Olkin y de esfericidad de Bartlett). Por la naturaleza ordinal de los datos, se utilizó el método de los mínimos cuadrados no ponderados, basado en la matriz de correlaciones policóricas (Freiberg Hoffmann, Stover, de la Iglesia \& Fernández Liporace, 2013; Lloret-Segura et al., 2014). Para determinar el número de factores se aplicó una estrategia analítica de dos pasos. Por un lado, se ejecutó un análisis paralelo optimizado, extrayendo aleatoriamente 500 submatrices e implementando el análisis de rango mínimo. Por otro lado, se efectuó la extracción de los factores sugeridos por el análisis paralelo, optando por la rotación oblicua Promin, ya que existía la presunción de que los elementos de la escala estaban relacionados (Baglin, 2014). El criterio para la selección de los ítems fue que pesaran 0,40 o más sobre el factor y que no saturaran sobre más de un factor al mismo tiempo (Lloret-Segura et al., 2014).

Sobre la otra mitad de la muestra se llevó a cabo un AFC con el método de máxima verosimilitud con la corrección robusta de Satorra-Bentler (S-B; Bentler, 2006). Para evaluar la bondad de ajuste del modelo se comprobó que el índice $\mathrm{S}-\mathrm{B} \chi^{2}$ sobre los grados de libertad $\left(\mathrm{S}-\mathrm{B} \chi^{2} / g l\right)$ fuera inferior a 3 , que el índice de bondad de ajuste de Jöreskog (GFI) y el índice de ajuste comparativo de Bentler-Bonett (CFI) alcanzaran valores iguales o superiores a 0,90 y que el valor de la raíz error cuadrático medio de aproximación (RMSEA) fuera inferior a 0,05 .

La invarianza configural se estableció a partir de un AFC multigrupo, ajustando el modelo sin restricciones en varones y mujeres. Para determinar la invarianza métrica, se realizó la prueba de diferencia de $\chi^{2}$, contrastando el $\chi^{2}$ obtenido en un modelo no constreñido con el $\chi^{2}$ correspondiente a un modelo con restricciones sobre los coeficientes de regresión. Si este valor es estadísticamente significativo, 
indica que las restricciones especificadas en el modelo más restrictivo no se sostienen, es decir, los dos modelos no son equivalentes entre los grupos (Dimitrov, 2010).

La consistencia interna del instrumento se estableció a partir del coeficiente de confiabilidad compuesta (CR, composite reliability) y del Coeficiente Alfa Ordinal (Gadermann, Guhn \& Zumbo, 2012). En ambos casos, valores por encima de 0,70 se consideran evidencia de adecuada consistencia interna (Gadermann et al., 2012).

La validez convergente-discriminante se determinó mediante el cálculo de la varianza media extraída (AVE, average variance extracted) y de su raíz cuadrada (Bagozzi \& Yi, 2012). El AVE permite estimar la varianza común entre los indicadores y su factor latente, considerando que valores superiores a 0,50 indican que más del $50 \%$ de la varianza del constructo es debida a sus indicadores. Por su parte, valores de la raíz cuadrada del AVE superiores a la correlación entre los factores latentes demuestra que cada constructo comparte más varianza con sus indicadores que con los demás. La validez concurrente se estableció a partir del cálculo de las relaciones entre los constructos estudiados. Dada la naturaleza ordinal de los datos, se utilizó el coeficiente de correlación de Spearman.

La validez predictiva e incremental se determinó a partir de un modelo de ecuaciones estructurales, asumiendo la satisfacción laboral como variable endógena (o dependiente), mientras que la pasión, el flow y la implicación en el trabajo desempeñaron el rol de variables exógenas (o independientes). En un primer modelo se calcularon los parámetros correspondientes a la regresión de la satisfacción laboral sobre las tres dimensiones del flow y sobre la implicación en el trabajo. En un segundo modelo se añadieron la pasión armoniosa y la pasión obsesiva. Las medidas de ajuste analizadas fueron el $R^{2}$ y el criterio de información de Akaike (AIC, Akaike Information Criterion).

El procesamiento y análisis de datos se realizó con los programas Factor, SPSS (versión 23.0) y EQS (versión 6.1).

\section{Resultados}

Resultados preliminares. El porcentaje de datos perdidos en cada ítem no superó el 5\%, respondiendo a una distribución aleatoria. Los valores faltantes fueron reemplazados mediante el método de estimación-maximización (Tabachnick \& Fidell, 2013). En la Tabla 1 se presentan los estadísticos descriptivos, índices de asimetría y curtosis e índices de discriminación correspondientes a los ítems de la Escala de Pasión por el Trabajo.

Tabla 1

Estadísticos Descriptivos, Índices de Asimetría y Curtosis y Coeficiente de homogeneidad corregido ( $r_{\text {-total }}$ ) correspondientes a los Ítems de la Escala de Pasión por el Trabajo $(N=452)$

\begin{tabular}{cccccc}
\hline Ítem & Media & $D E$ & Asimetría & Curtosis & $r_{\text {i.total }}$ \\
\hline 1 & 3,90 & 1,18 & 1,28 & 0,89 & 0,54 \\
2 & 3,57 & 1,03 & $-0,93$ & 0,54 & 0,50 \\
3 & 3,29 & 1,05 & $-0,91$ & 0,10 & 0,43 \\
4 & 3,61 & 1,09 & 0,98 & 0,56 & 0,51 \\
5 & 3,35 & 1,04 & $-0,89$ & 0,35 & 0,50 \\
6 & 3,69 & 1,06 & $-0,99$ & 0,91 & 0,49 \\
7 & 2,95 & 1,24 & $-0,25$ & $-1,14$ & 0,53 \\
8 & 2,36 & 1,20 & 0,61 & $-0,36$ & 0,60 \\
9 & 2,30 & 1,05 & 0,44 & $-0,47$ & 0,58 \\
10 & 2,58 & 1,07 & 0,64 & $-0,15$ & 0,57 \\
11 & 2,76 & 1,10 & 0,62 & $-0,35$ & 0,53 \\
12 & 2,46 & 1,09 & 1,10 & 0,62 & 0,50 \\
13 & 2,82 & 1,14 & 0,71 & 0,57 & 0,49 \\
14 & 2,79 & 1,11 & 1,11 & 0,50 & 0,41 \\
\hline
\end{tabular}


Como puede observarse en la Tabla 1, en ningún caso los valores de asimetría y curtosis superaron el límite establecido, variaron entre $-0,99$ a 1,28 (Mediana $=0,61)$ y entre $-1,14$ a 0,91 (Mediana $=0,43)$, respectivamente. Las correlaciones ítem-total fueron directas, variando entre 0,41 y 0,60. Los resultados de la prueba de Harman permiten afirmar que los datos no presentan sesgos debidos al método común, desde el momento en que la extracción de un único factor aglutinó solo un 21,74\% de la varianza total. El AFC mostró que el modelo de medida unifactorial mostró un ajuste inadecuado $\left(\mathrm{S}-\mathrm{B} \chi^{2} / g l=5,06, \mathrm{GFI}=0,78, \mathrm{CFI}=0,77\right.$, RMSEA $=0,10[0,08,0,11])$. En contraposición, el modelo heptafactorial presentó un ajuste satisfactorio a la matriz de varianzas y covarianzas empírica $\left(\mathrm{S}-\mathrm{B} \chi^{2} / g l=2,16, \mathrm{GFI}=0,91\right.$, CFI $=0,93$, RMSEA $=0,04[0,03$, 0,05]). El cómputo del criterio de información de Akaike para ambos modelos también proporcionó evidencia favorable al modelo de medida de siete factores correlacionados $\left(\mathrm{AIC}_{\text {modelo }}\right.$ uni-factorial $=537,48$, $\mathrm{AIC}$ modelo heptafactorial $=462,15)$.

Evidencias de validez de constructo. La prueba de esfericidad de Bartlett fue significativa, $\chi^{2}(91$, $226)=1694,29, p<0,001)$ y el índice de adecuación muestral de Kaiser-Meyer-Olkin arrojó un valor de 0,91. Los resultados del análisis paralelo (Tabla 2) indican que los autovalores empíricos correspondiente al primer y segundo factor real se encuentran por encima del promedio y del percentil 95 de los autovalores aleatorios, sugiriendo, de este modo, dos dimensiones subyacentes. El porcentaje global de varianza común explicada por los factores extraídos fue de 61,14.

Tabla 2

Autovalores Reales y Promedio de Autovalores Aleatorios según Análisis Paralelo de Horn

\begin{tabular}{cccc}
\hline Variable & Autovalores reales & $\begin{array}{c}\text { Media autovalores } \\
\text { aleatorios }\end{array}$ & $\begin{array}{c}\text { Percentil 95 de } \\
\text { autovalores aleatorios }\end{array}$ \\
\hline 1 & $6,06^{*}$ & 1,43 & 1,53 \\
2 & $2,65^{*}$ & 1,33 & 1,40 \\
3 & 0,90 & 1,25 & 1,31 \\
4 & 0,79 & 1,18 & 1,23 \\
5 & 0,54 & 1,12 & 1,17 \\
6 & 0,51 & 1,06 & 1,10 \\
7 & 0,45 & 1,00 & 1,05 \\
8 & 0,41 & 0,95 & 0,99 \\
9 & 0,40 & 0,90 & 0,94 \\
10 & 0,35 & 0,85 & 0,90 \\
11 & 0,28 & 0,80 & 0,84 \\
12 & 0,22 & 0,75 & 0,79 \\
13 & 0,21 & 0,69 & 0,74 \\
14 & 0,15 & 0,62 & 0,68 \\
\hline
\end{tabular}

Nota. Con asterisco se señalan los factores a retener.

La distribución de los reactivos coincidió con las dimensiones propuestas originalmente, por las que fueron rotuladas Pasión armoniosa y Pasión Obsesiva. En línea con las recomendaciones de la literatura especializada (Lloret-Segura et al., 2014), en la Tabla 3 se informa tanto la matriz de configuración con las saturaciones factoriales de cada ítem en cada factor, como la matriz de estructura, que contiene las correlaciones entre las variables observadas y las variables latentes. 
Tabla 3

Matriz de Estructura y Matriz de Configuración Correspondiente a los Ítems de la Escala de Pasión por el Trabajo $(N=226)$

\begin{tabular}{|c|c|c|c|c|}
\hline \multirow{2}{*}{$\begin{array}{l}\text { Subescala e ítem } \\
\text { Pasión armoniosa }\end{array}$} & \multicolumn{2}{|c|}{$\begin{array}{l}\text { Matriz de } \\
\text { estructura }\end{array}$} & \multicolumn{2}{|c|}{$\begin{array}{l}\text { Matriz de } \\
\text { configuración }\end{array}$} \\
\hline & I & II & I & II \\
\hline 1. Este trabajo me permite vivir diversas experiencias & 0,80 & & 0,70 & \\
\hline 2. Las cosas que aprendo en este trabajo hacen que lo valore más & 0,81 & & 0,85 & \\
\hline 3. Este trabajo está en equilibrio con otras actividades de mi vida & 0,72 & & 0,68 & \\
\hline 4. Este trabajo refleja las cualidades que valoro en mí & 0,78 & & 0,77 & \\
\hline 5. Estoy completamente implicado con este trabajo & 0,77 & & 0,80 & \\
\hline 6. Este trabajo me permite vivir experiencias inolvidables & 0,83 & & 0,88 & \\
\hline 7. Este trabajo es una pasión que todavía logro controlar & 0,68 & & 0,66 & \\
\hline \multicolumn{5}{|l|}{ Varianza explicada: $45 \%$} \\
\hline \multicolumn{5}{|l|}{ Pasión obsesiva } \\
\hline 1. No puedo vivir sin este trabajo & & 0,77 & & 0,77 \\
\hline 2. Dependo emocionalmente de este trabajo & & 0,78 & & 0,81 \\
\hline 3. Me cuesta controlar la necesidad de realizar este trabajo & & 0,80 & & 0,84 \\
\hline 4. El impulso es tan fuerte, que no puedo dejar de hacer este trabajo & & 0,71 & & 0,69 \\
\hline 5. Tengo un sentimiento casi obsesivo por este trabajo & & 0,85 & & 0,85 \\
\hline 6. Mi estado de ánimo depende de este trabajo & & 0,72 & & 0,66 \\
\hline 7. No puedo concebir mi vida sin este trabajo & & 0,81 & & 0,80 \\
\hline Varianza explicada: $16,14 \%$ & & & & \\
\hline
\end{tabular}

Siguiendo los lineamientos de la estrategia de modelización confirmatoria, el ajuste del modelo derivado del AFE fue analizado a partir de una serie de estadísticos. Los valores obtenidos en cada índice demuestran que un modelo integrado por dos factores latentes correlacionados con 14 ítems como indicadores observables y sus respectivos errores de medición no difiere de la matriz de datos $\left(\mathrm{S}-\mathrm{B} \chi^{2} / g l=1,85, \mathrm{GFI}=0,90, \mathrm{CFI}=0,94\right.$, RMSEA $=0,04[0,03,0,05])$. Las cargas factoriales oscilaron entre 0,57 y $0,82(p<0,001)$ para el factor Pasión armoniosa y entre 0,66 y 0,84 $(p<0,001)$ para el factor Pasión Obsesiva. El modelo de medida final correspondiente a la escala se muestra en la Figura 1.

El AFC multigrupo determinó que la estructura factorial era adecuada cuando ambos grupos (varones y mujeres) se probaron juntos y $\sin$ restricciones $\left(\mathrm{S}-\mathrm{B} \chi^{2} / g l=2,18, \mathrm{GFI}=0,90, \mathrm{CFI}=0,90, \mathrm{RMSEA}=0,03\right.$ $[0,02,0,04])$. La prueba de diferencia entre el $\chi^{2}$ obtenido en un modelo no constreñido y un modelo alternativo con restricciones sobre los coeficientes de regresión no alcanzó valores estadísticamente significativos, $\left.\chi^{2}(12, \boldsymbol{N}=452)=7,8, p=0,802\right)$ indicando, entonces, la no existencia de diferencias entre los grupos. Tales resultados proporcionaron evidencia a favor de la invariancia configural y métrica de la versión argentina de la Escala de Pasión por el Trabajo.

Evidencias de confiabilidad. El factor Pasión armoniosa obtuvo un alfa ordinal de 0,79 y un $C R$ de 0,80, en tanto que el factor Pasión Obsesiva superó levemente esos valores, 0,80 y 0,81, respectivamente. Tales resultados demuestran que la versión adaptada del instrumento cuenta con adecuada consistencia interna y confiabilidad compuesta. 


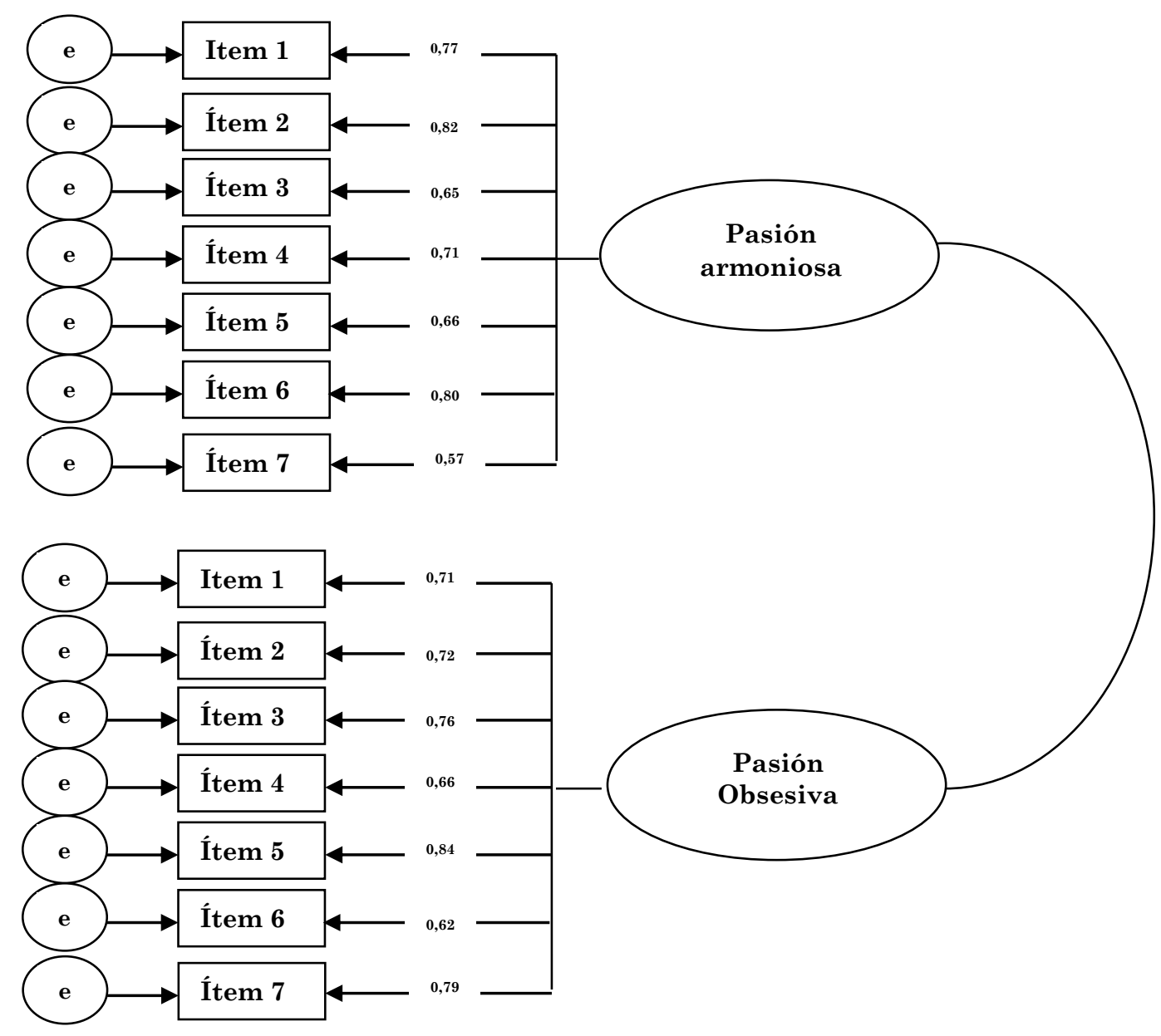

Figura 1. Modelo de medida correspondiente a la adaptación de la Escala de Pasión por el Trabajo $(n=226)$.

Evidencias de validez. En la Tabla 4 se muestran los coeficientes de correlación de Spearman, las correlaciones policóricas entre los factores latentes, y los valores correspondientes al índice AVE y a su raíz cuadrada.

Tabla 4

Correlaciones entre Factores Latentes y Observados, Índice de Varianza Media Extraída (AVE) y Raíz Cuadrada del AVE Correspondientes a las Variables bajo Estudio $(N=452)$

\begin{tabular}{lccccccccc}
\hline \multicolumn{1}{c}{ Variable } & 1 & 2 & 3 & 4 & 5 & 6 & 7 & AVE & VAVE \\
\hline 1. Pasión armoniosa & - & $0,40^{* *}$ & $0,64^{* *}$ & $0,69^{* *}$ & $0,50^{* *}$ & $0,64^{* *}$ & $0,60^{* *}$ & 0,77 & 0,87 \\
2. Pasión obsesiva & $0,45^{* *}$ & - & 0,13 & $0,62^{* *}$ & $0,47^{* *}$ & $0,30^{* *}$ & $0,45^{* *}$ & 0,80 & 0,89 \\
3. Satisfacción laboral & $0,38^{* *}$ & 0,18 & - & $0,55^{* *}$ & $0,42^{* *}$ & $0,67^{* *}$ & $0,62^{* *}$ & 0,82 & 0,90 \\
4. Implicación laboral & $0,72^{* *}$ & $0,57^{* *}$ & $0,50^{* *}$ & - & $0,51^{* *}$ & $0,56^{* *}$ & $0,59^{* *}$ & 0,73 & 0,85 \\
5. Absorción & $0,59^{* *}$ & $0,62^{* *}$ & $0,37^{* *}$ & $0,75^{* *}$ & - & $0,45^{* *}$ & $0,42^{* *}$ & 0,76 & 0,87 \\
6. Disfrute & $0,34^{* *}$ & $0,39^{* *}$ & $0,75^{* *}$ & $0,62^{* *}$ & $0,50^{* *}$ & - & $0,57^{* *}$ & 0,71 & 0,84 \\
7. Motivación & $0,57^{* *}$ & $0,50^{* *}$ & $0,78^{* *}$ & $0,61^{* *}$ & $0,63^{* *}$ & $0,65^{* *}$ & - & 0,73 & 0,85 \\
\hline
\end{tabular}

Nota. ${ }^{* *} p<0,001$. Bajo la diagonal se informan las correlaciones policóricas entre los factores latentes. Sobre la diagonal se presentan los coeficientes de correlación de Spearman. 
De la Tabla 4 se desprende que las dos formas de pasión se asociaron entre sí y en la dirección esperada. En lo que respecta a sus vinculaciones con el resto de las variables analizadas, la pasión armoniosa correlacionó fuertemente con el flow, la implicación en el trabajo y la satisfacción laboral, en tanto que la pasión obsesiva evidenció fuertes asociaciones con la implicación en el trabajo y el flow, no encontrándose correlación con la satisfacción laboral. Las raíces cuadradas del AVE correspondiente a los factores Pasión armoniosa y Pasión Obsesiva fueron mayores que la correlación entre estos y los restantes constructos. Del mismo modo, el valor del AVE se ubicó por encima del mínimo recomendado.

Paralelamente, el análisis de ecuaciones estructurales mostró que todos los parámetros fueron estadísticamente significativos para el primer modelo. En efecto, las dimensiones de disfrute, $\Upsilon=0,31, p<0,001$, motivación intrínseca, $\Upsilon=0,22, p<0,031$ y absorción, $\Upsilon=0,20, p<0,020$, correspondientes al flow, así como la implicación en el trabajo, $\Upsilon=0,27, p<0,032$, realizaron una contribución a la satisfacción, explicando en conjunto el $46,20 \%$ de su varianza. Al añadir la pasión armoniosa y la pasión obsesiva, se registró un aumento del $5,8 \%$ en la varianza explicada de la satisfacción laboral, $\left(\Delta R^{2}=0,520-0,462=0,058\right)$. La inspección de los coeficientes de regresión permite señalar que la pasión armoniosa realiza una contribución más elevada, $\Upsilon=0,32, p<0,001$, que la pasión obsesiva, $\Upsilon=0,17, p<0,025$. Sumado al incremento en $R^{2}$, el valor del $\mathrm{AIC}\left(\mathrm{AIC}_{\text {primer modelo }}=85,9 ; \mathrm{AIC}_{\text {segundo modelo }}=78,2\right)$ muestra que la varianza explicada por el modelo que incluye a la pasión por el trabajo aporta mejor información que el primer modelo.

En conjunto, estos resultados proporcionan evidencia favorable sobre la validez concurrente, convergente, discriminante, predictiva e incremental de la Escala de Pasión por el Trabajo en su versión argentina.

\section{Discusión}

La investigación realizada tuvo como objetivos adaptar y validar la Escala de Pasión por el Trabajo (Vallerand et al., 2003) para su empleo en Argentina, así como analizar sus propiedades psicométricas. En lo que hace a su estructura interna, la literatura publicada muestra un consenso generalizado en concebir a la pasión como un constructo bidimensional (Forest et al., 2011; Perrewé et al., 2014; Vallerand, 2015). En línea con tales hallazgos, los análisis factoriales desarrollados en el presente estudio ratificaron que el modelo de medida que mejor capta la esencia del concepto es aquel compuesto por dos factores latentes, uno correspondiente a la pasión obsesiva y otro referente a la pasión armoniosa. De este modo, los resultados obtenidos concuerdan con las conclusiones de diversas validaciones desarrolladas hasta el momento (Orgambídez-Ramos et al., 2014; van der Knaap \& Steensma, 2015; Zito \& Colombo, 2017).

A su vez, los resultados señalan la invarianza del instrumento, tanto a nivel configural como métrico. Tales hallazgos coinciden con los informados por otras validaciones (Orgambídez-Ramos et al., 2014; Zito \& Colombo, 2017). Paralelamente, sintonizan con los resultados de Marsh et al. (2013), quienes demostraron la invarianza del instrumento a distintos niveles (configural, métrica y estricta), en dos idiomas (inglés y francés) y en relación a diversas actividades (trabajo, educación, deporte y placer), y entre hombres y mujeres. Vale decir, ya sea entre varones y mujeres, franceses y anglosajones, así como entre las diversas actividades apasionantes, tanto la configuración del modelo de medida como las saturaciones factoriales correspondientes a cada ítem y los valores interceptales de los factores son equivalentes. En suma, los hallazgos del presente estudio se integran a la evidencia disponible, permitiendo concluir que la escala podrá ser utilizada con la misma validez y confiabilidad tanto con muestras de varones como de mujeres.

Del presente estudio se desprende también que la escala posee una adecuada validez convergentediscriminante, dado que la varianza capturada por los factores identificados es mayor que la debida a los errores de medida y se puede explicar adecuadamente a través de los indicadores elegidos (Bagozzi \& Yi, 2012). Asimismo, la raíz cuadrada del AVE fue mayor que la correlación al cuadrado entre cada dimensión y las demás, ratificando la independencia empírica de los conceptos analizados. En línea con tales resultados, un reciente estudio (Salessi \& Omar, 2018a) ha demostrado que los constructos pasión por el trabajo, flow e implicación en el trabajo cumplen el criterio de validez discriminante, puesto que sus respectivas mediciones son empíricamente únicas y cada uno de ellos representa un fenómeno que los otros constructos no capturan.

En lo que respecta a la validez concurrente, el análisis de correlaciones efectuado indica que la pasión armoniosa y la pasión obsesiva correlacionan directamente entre sí, coincidiendo con la evidencia empírica publicada (Orgambídez-Ramos et al., 2014; Serrano-Fernández et al., 2017; Vallerand et al., 2003; van der Knaap \& Steensma, 2015; Zito \& Colombo, 2017). Del mismo modo, ambos tipos de pasión se asocian en las 
direcciones esperadas con el resto de los constructos estudiados. En este sentido, las vinculaciones directas entre la pasión armoniosa y la pasión obsesiva con la implicación en el trabajo se encuentran en línea con numerosos estudios (Birkeland \& Buch, 2015; Houlfort, Vallerand \& Laframboise, 2015; Loriol, 2017; Serrano-Fernández et al., 2017). De acuerdo a sus hallazgos, los trabajadores apasionados (sea armónicamente, sea obsesivamente) se encuentran cognitiva y emocionalmente absorbidos y comprometidos por su trabajo, concibiéndolo como un dominio central en sus vidas y un aspecto crítico para su autoestima y autoimagen.

En cuanto al flow, ambos tipos de pasión mostraron un patrón de correlaciones directas con dicho constructo. Si bien las vinculaciones con la pasión armoniosa se encuentran en línea con la teoría y la evidencia empírica (Carpentier, Mageau, \& Vallerand, 2012; Curran et al., 2015; Lavigne, Forest \& Crevier-Braud, 2012), los resultados obtenidos en torno a la pasión obsesiva resultan novedosos. Tales hallazgos, lejos de ser contradictorios, son consistentes con la esencia misma del constructo, ya que los individuos obsesivamente apasionados se encuentran abocados a su trabajo. Además, se encuentran en sintonía con recientes hallazgos (Zito \& Colombo, 2017), de los que también se desprenden correlaciones directas entre el flow y la pasión obsesiva. Para comprender tales resultados, basta considerar que los altos montos de esfuerzo y dedicación invertidos por estos trabajadores en la ejecución de su tarea serían capaces de explicar la absorción y motivación intrínseca experimentada en su trabajo, así como la posibilidad de cierto disfrute y satisfacción mientras estén involucrados en él. De aquí también resultan comprensibles los hallazgos de otras investigaciones (Carpentier et al., 2012; Curran et al., 2015; Lavigne et al., 2012) que han observado que la pasión obsesiva se asocia a pensamientos rumiantes y menores experiencias de flow cuando el individuo no está trabajando, sino que se encuentra participando de otras actividades diferentes que no considera apasionantes.

Los resultados del presente estudio también proporcionan evidencia favorable sobre la validez predicativa e incremental de la pasión respecto del flow y de la implicación en el trabajo. En efecto, la pasión armoniosa y obsesiva realizan un incremento a la varianza explicada de la satisfacción laboral, más allá de los efectos del disfrute, la motivación intrínseca, la absorción y la implicación. Si bien del análisis correlacional ejecutado se desprende que la pasión obsesiva no se asoció con la satisfacción laboral, en el análisis de ecuaciones estructurales sí emergió como un predictor relevante. Tales hallazgos, en lugar de ser incongruentes, pueden ser explicados en el marco de lo que se denomina supresión clásica (McFatter, 1979). En este sentido, un efecto de supresión ocurre cuando se produce un aumento en la predicción de un criterio al incluir una variable predictora no relacionada a él, pero sí a otro u otros predictores. Dicha variable (en este caso, la pasión obsesiva) recibe el nombre de supresor, porque suprime o elimina varianza irrelevante, aumentando la proporción de la varianza explicada por los predictores (Maassen \& Bakker, 2001), situación que se observa en el presente estudio.

En línea con tales hallazgos, diversas investigaciones comprueban el aporte sustancial atribuible a la pasión en la explicación de diversas actitudes y comportamientos. En este sentido, por ejemplo, Birkeland y Buch (2015) encontraron que, luego de controlar los efectos de la adicción al trabajo y del engagement, la pasión por el trabajo en sus dos vertientes predice el burnout. Del mismo modo, Houlfort et al. (2014) hallaron que la pasión armoniosa y obsesiva explican las intenciones de renunciar y la satisfacción de los empleados más allá de sus niveles de motivación en el trabajo. Paralelamente, Thorgren, Wincent y Sirén (2013) señalaron que la pasión contribuye incrementalmente en la predicción de la satisfacción laboral.

En conjunto, los resultados aquí encontrados sintonizan con la evidencia publicada, demostrando tanto la validez del instrumento analizado como del constructo pasión por el trabajo.

\section{Limitaciones, Fortalezas e Implicancias Prácticas del Estudio}

Entre las principales limitaciones de la investigación realizada hay que citar la representatividad de la muestra, ya que por haber trabajado con muestras por conveniencia se ve dificultada la generalización de los resultados a la población en su conjunto. Además, como no se ha verificado la estabilidad de las dimensiones a lo largo del tiempo, sería recomendable explorar la confiabilidad test-retest de la escala. Por último, hay que remarcar que como se trata de un instrumento de autoinforme, las respuestas podrían verse contaminadas, tanto por la deseabilidad social como por otros componentes subjetivos, fundamentalmente en lo que respecta a la percepción de la pasión obsesiva.

Más allá de estas limitaciones, el trabajo realizado constituye la primera tentativa de operacionalizar el constructo pasión por el trabajo en Argentina, por lo que representa una contribución genuina a la 
investigación en la temática. La validación efectuada abre la necesidad de llevar a cabo nuevos estudios instrumentales en otros contextos laborales y culturales tales como, por ejemplo, sobre muestras ocupacionales específicas, sobre muestras de trabajadores mal remunerados, en poblaciones rurales, etc. Esa sería una vía idónea para verificar la validez transcultural del instrumento aquí presentado.

Finalmente, disponer de un instrumento válido y confiable para medir la pasión conlleva numerosos beneficios prácticos. La evidencia empírica acumulada en estos años demuestra que la pasión se asocia a diversas actitudes y comportamientos relevantes, que incluyen desde el bienestar ocupacional hasta el enriquecimiento trabajo-familia. Sea en calidad de protagonista o como actor de reparto que se enlaza a otros factores para influir indirectamente, la pasión por el trabajo ha demostrado ser un constructo multifacético capaz de desempeñar diversos roles. A su vez, la investigación contemporánea (Perrewé et al., 2014; Vallerand, 2015; Zigarmi et al., 2009) muestra que la variabilidad interindividual no se debe tanto a las diferencias en rasgos fijos y estables, sino a características maleables y abiertas al cambio, lo que posibilita intervenir de cara a su desarrollo. Frente a este panorama, las organizaciones preocupadas por garantizar el bienestar de sus miembros e incrementar su productividad, deberían concentrarse en la creación de un entorno capaz de contribuir al florecimiento de la pasión, principalmente, de la pasión armoniosa, dada la numerosa evidencia que subraya sus consecuencias benéficas (Curran et al., 2015; Dubreuil et al., 2014; Houlfort et al., 2014; Vallerand, 2015). En este sentido, promover una cultura que afiance los lazos interpersonales y fomente un sentido de pertenencia y espíritu de grupo, formar a quienes ocupen posiciones clave como líderes transformacionales que inspiren a sus seguidores y diseñar puestos que brinden mayor autonomía en la planificación del trabajo y fortalezcan el sentido de autoeficacia, serían intervenciones favorecedoras de la pasión en su vertiente positiva.

\section{Referencias}

Anderson, J. C. \& Gerbing, D. W. (1982). Some methods for respecifying measurement models to obtain unidimensional construct measurement. Journal of Marketing Research, 19, 453-460. https://doi.org/10.1177/002224378201900407

Andreassen, C. S. (2014). Workaholism: An overview and current status of the research. Journal of Behavioral Addictions, 3, 1-11. https://doi.org/10.1556/jba.2.2013.017

Ato, M., López, J. J. \& Benavente, A. (2013). Un sistema de clasificación de los diseños de investigación en psicología. Anales de Psicología, 29, 1038-1059. https://doi.org/10.6018/analesps.29.3.178511

Bakker, A. B. (2008). The Work-Related Flow Inventory: Construction and initial validation of the WOLF. Journal of Vocational Behavior, 72, 400-414. https://doi.org/10.1016/j.jvb.2007.11.007

Bakker, A. \& Albrecht, S. (2018). Work engagement: current trends. Career Development International, 23(1), 4-11, https://doi.org/10.1108/CDI-11-2017-0207

Baglin, J. (2014). Improving your exploratory factor analysis for ordinal data: A demonstration using FACTOR. Practical Assessment, Research \& Evaluation, 19(5), 1-15. Extraído de http://pareonline.net/getvn.asp?v=19\&n=5

Bagozzi, R. P. \& Yi, Y. (2012). Specification, evaluation, and interpretation of structural equation models. Journal of the Academy of Marketing Science, 40, 8-34. https://doi.org/10.1007/s11747-011-0278-x

Bentler, P. M. (2006). EQS 6 structural equations program manual. Encino, CA: Multivariate Software.

Birkeland, I. K. \& Buch, R. (2015). The dualistic model of passion for work: Discriminate and predictive validity with work engagement and workaholism. Motivation and Emotion, 39, 392-408. https://doi.org/10.1007/s11031-014-9462-x

Carpentier, J., Mageau. G. A. \& Vallerand, R. J. (2012). Ruminations and flow: Why do people with a more harmonious passion experience higher well-being? Journal of Happiness Studies, 13, 501-518. https://doi.org/10.1007/s10902-011-9276-4

Curran, T., Hill, A. P., Appleton, P. R., Vallerand, R. J. \& Standage, M. (2015). The psychology of passion: A meta-analytical review of a decade of research on intrapersonal outcomes. Motivation and Emotion, 39, 631-655. https://doi.org/10.1007/s11031-015-9503-0

Dimitrov, D. M. (2010). Testing for factorial invariance in the context of construct validation. Measurement and Evaluation in Counseling and Development, 43, 121-149. https://doi.org/10.1177/0748175610373459

Dubreuil, P., Forest, J. \& Courcy, F. (2014). From strengths use to work performance: The role of harmonious passion, subjective vitality, and concentration. The Journal of Positive Psychology, 9, 335-349. https://doi.org/10.1080/17439760.2014.898318

Engeser, S. \& Baumann, N. (2016). Fluctuation of flow and affect in everyday life: A second look at the paradox of work. Journal of Happiness Studies, 17, 105-124. https://doi.org/10.1007/s10902-014-9586-4

Forest, J., Mageau, G. A., Sarrazin, C. \& Morin, E. M. (2011). "Work is my passion": The different affective, behavioural, and cognitive consequences of harmonious and obsessive passion toward work. Canadian Journal of Administrative Sciences, 28, 27-40. https://doi.org/10.1002/cjas.170

Freiberg Hoffmann, A., Stover, J. B., de la Iglesia, G. \& Fernández Liporace, M. (2013). Correlaciones policóricas y tetracóricas en estudios factoriales exploratorios y confirmatorios. Ciencias Psicológicas, 7, 151-164. Extraído de http://www.scielo.edu.uy/scielo.php?script=sci_arttext\&pid=S1688-42212013000200005

Gadermann, A. M., Guhn, M. \& Zumbo, B. D. (2012). Estimating ordinal reliability for Likert-tipe and ordinal item response data: A conceptual, empirical, and practical guide. Practical Assessment, Research \& Evaluation, 17(3), 1-13. Extraído de http://pareonline.net/pdf/v17n3.pdf

Hair Jr., J. E., Black, W. C., Babin, B. J., Anderson, R. E. \& Tatham, R. L. (2010). Multivariate data analysis (7a ed.). Upper Saddle River, NJ: Pearson Education.

Houlfort, N. Philippe, F. L., Vallerand, R. J. \& Ménard, J. (2014). On passion and heavy work investment: Personal and organizational outcomes. Journal of Managerial Psychology, 29, 25-45. https://doi.org/10.1108/JMP-06-2013-0155 
Kanungo, R. N. (1982). Measurement of job and work involvement. Journal of Applied Psychology, 67, 341-349. https://doi.org/10.1037/0021-9010.67.3.341

Lajom, J. A. L., Amarnani, R. K., Restubog, S. L. D., Bordia, P. \& Tang, R. L. (2018). Dualistic passion for work and its impact on career outcomes: Scale validation and nomological network. Journal of Career Assessment, 26, 631-648. https://doi.org/10.1177/1069072717723096

Lalande, D., Vallerand, R. J., Lafrenière, M. -A. K., Verner-Filion, J., Laurent, F. -A., Forest, J. \& Paquet, Y. (2017). Obsessive passion: A compensatory response to unsatisfied needs. Journal of Personality, 85, 163-178. https://doi.org/10.1111/jopy.12229

Lavigne, G. L., Forest, J. \& Crevier-Braud, L. (2012). Passion at work and burnout: A two-study test of the mediating role of flow experiences. European Journal of Work \& Organizational Psychology, 21, 518-546. https://doi.org/10.1080/1359432X.2011.578390

Lloret-Segura, S., Ferreres-Traver, A., Hernández-Baeza, A. \& Tomás-Marco, I. (2014). El análisis factorial exploratorio de los ítems: una guía práctica, revisada y actualizada. Anales de Psicología, 30, 1151-1169. https://doi.org/10.6018/analesps.30.3.199361

Loriol, M. (2017). The collective regulation of smac workers' passion and involvement. Sociologia del Lavoro, 145, 168-183. https://doi.org/10.3280/SL2017-145010

Maassen, G. H. \& Bakker, A. B. (2001). Suppressor variables in path models: Definitions and interpretations. Sociological Methods \& Research, 30, 241-270. https://doi.org/10.1177/0049124101030002004

Macdonald, S. \& MacIntyre, P. (1997). The Generic Job Satisfaction Scale: Scale development and its correlates. Employee Assistance Quarterly, 13(2), 1-16. https://doi.org/10.1300/j022v13n02_01

Mageau, G. A., Carpentier, J. \& Vallerand, R. J. (2011). The role of self-esteem contingencies in the distinction between obsessive and harmonious passion. European Journal of Social Psychology, 41, 720-729. https://doi.org/10.1002/ejsp.798

Marsh, H. W., Vallerand, R. J., Lafrenière, M. -A. K., Parker, P., Morin, A. J. S., Carbonneau, N. ... Paquet, Y. (2013). Passion: Does one scale fit all? Construct validity of two-factor passion scale and psychometric invariance over different activities and languages. Psychological Assessment, 25, 796-809. https://doi.org/10.1037/a0032573

Martins, M., Araújo, A. M. \& Almeida, L. S. (2014). Paixão pelo trabalho e competências de gestão interpessoal: validação de escalas para a sua avaliação junto de gestores empresariais [Pasión por el trabajo y competencias de gestión interpersonal: validación de escalas para su evaluación en gerentes de empresas]. Psychologica da Universidade de Coimbra, 57, 61-76. https://doi.org/10.14195/1647-8606_57_1_4

McFatter, R. M. (1979). The use of structural equation models in interpreting regression equations including suppressor and enhancer variables. Applied Psychological Measurement, 3, 123-135 https://doi.org/10.1177/014662167900300113

Meyer, J. P. (Ed.) (2016). Handbook of employee commitment. Cheltenham, Reino Unido: Edward Elgar. https://doi.org/10.4337/9781784711740

Muñiz, J., Elosua, P. \& Hambleton, R. K. (2013). Directrices para la traducción y adaptación de los tests: segunda edición. Psicothema, 25, 151-157. https://doi.org/10.7334/psicothema2013.24

Orgambídez-Ramos, A., Borrego-Alés, Y. \& Gonçalves, G. (2014). Passionate workers: A Spanish adaptation of the Passion Scale. Revista de Psicología del Trabajo y de las Organizaciones, 30, 43-48. https://doi.org/10.1016/j.rpto.2014.06.005

Pérez-Gil, J. A., Chacón Moscoso, S. \& Moreno Rodríguez, R. (2000). Validez de constructo: el uso del análisis factorial exploratorioconfirmatorio para obtener evidencias de validez. Psicothema, 12(Suplemento 2), 442-446. Extraído de http://www.psicothema.com/psicothema.asp?id=601

Perrewé, P. L., Hochwarter, W. A., Ferris, G. R., McAllister, C. P. \& Harris, J. N. (2014). Developing a passion for work passion: Future directions on an emerging construct. Journal of Organizational Behavior, 35(1), 145-150. https://doi.org/10.1002/job.1902

Podsakoff, P. M., MacKenzie, S. B. \& Podsakoff, N. P. (2012). Sources of method bias in social science research and recommendations on how to control it. Annual Review of Psychology, 63, 539-569. https://doi.org/10.1146/annurev-psych-120710-100452

Salessi, S. \& Omar, A. (2018a). Pasión por el Trabajo: evidencias de validez discriminante, predictiva e incremental. Psicologia: Ciência e Profissão, 38(3), 1-15. https://doi.org/10.1590/1982-37030004752017

Salessi, S. \& Omar, A. (2018b). Cuestionario de implicación en el trabajo: Propiedades psicométricas de su adaptación en Argentina. Revista Mexicana de Psicología, 35(2), 179-192. Extraído de https://www.redalyc.org/jatsRepo/2430/243059346007/html/index.html

Salessi, S. \& Omar, A. (2018c). Inventario de flow en el trabajo: evidencias de validez de estructura interna y criterio. Avaliação Psicológica, 17(3), 378-388. https://doi.org/10.15689/ap.2018.1703.14741.11.

Serrano-Fernández, M. J., Boada-Grau, J. Gil-Ripoll, C. \& Vigil-Colet, A. (2017). Spanish adaptation of the Passion toward Work Scale (PTWS). Anales de Psicología, 33, 403-410. https://doi.org/10.6018/analesps.33.2.240521

Tabachnick, B. G. \& Fidell, L. S. (2013). Using multivariate statistics (6 ${ }^{\text {a }}$ ed.). Boston, MA: Pearson.

Taris, T., van Beek, I. \& Schaufeli, W. (2014). The beauty versus the beast: On the motives of engaged and workaholic employees. En I. Harpaz \& R. Snir (Eds.), Heavy work investment: Its nature, sources, outcomes, and future directions (pp. 121-139). New York, NY: Routledge/Taylor \& Francis.

Thorgren, S., Wincent, J. \& Sirén, C. (2013). The influence of passion and work-life thoughts on work satisfaction. Human Resource Development Quarterly, 24, 469-492. https://doi.org/10.1002/hrdq.21172

Vallerand, R. J. (2015). The psychology of passion: A dualistic model. New York, NY: Oxford University Press.

Vallerand, R. J., Blanchard, C., Mageau, G. A., Koestner, R., Ratelle, C., Léonard, M. ... Marsolais, J. (2003). Les passions de l'âme: On obsessive and harmonious passion [Las pasiones del alma: sobre la pasión obsesiva y armoniosa]. Journal of Personality and Social Psychology, 85, 756-767. https://doi.org/10.1037/0022-3514.85.4.756

Vallerand, R. J. \& Houlfort, N. (2003). Passion at work: Toward a new conceptualization. En S. W. Gilliland, D. D. Steiner \& D. P. Skarlicki (Eds.), Emerging perspectives on values in organizations (pp. 175-204). Charlotte, NC: Information Age Publishing.

Vallerand, R. J. \& Verner-Filion, J. (2013). Making peoples' life most worth living: On the importance of passion for positive psychology. Terapia Psicológica, 31, 35-48. https://doi.org/10.4067/s0718-48082013000100004

van der Knaap, M. \& Steensma, H. (2015). Validatie van de Dutch Passion Scale op basis van het dualistische model van passie [Validación holandesa de la escala de pasión basada en el modelo dualista de la pasión]. Gedrag \& Organisatie, 28(1), 46-60.

Zigarmi, D., Nimon, K., Houson, D., Witt, D. \& Diehl, J. (2009). Beyond engagement: Toward a framework and operational definition for employee work passion. Human Resource Development Review, 8, 300-326. https://doi.org/10.1177/1534484309338171

Zito, M. \& Colombo, L. (2017). The Italian version of the Passion for Work Scale: First psychometric evaluations. Journal of Work and Organizational Psychology, 33, 47-53. https://doi.org/10.1016/j.rpto.2017.01.003 
Anexo

\section{Escala Pasión por el Trabajo (Adaptación Argentina)}

A continuación encontrará una serie de afirmaciones sobre el trabajo. Por favor, luego de leer cada oración, marque la opción que mejor representa sus sentimientos y pensamientos habituales. El significado de cada número es el siguiente:

\begin{tabular}{|c|c|c|c|c|}
\hline $\begin{array}{c}\text { Totalmente en } \\
\text { desacuerdo }\end{array}$ & En desacuerdo & $\begin{array}{c}\text { Ni acuerdo } \\
\text { ni en desacuerdo }\end{array}$ & De acuerdo & $\begin{array}{c}\text { Totalmente de } \\
\text { acuerdo }\end{array}$ \\
\hline 1 & 2 & 3 & 4 & 5 \\
\hline
\end{tabular}

\begin{tabular}{|c|c|c|c|c|c|c|}
\hline 1 & Este trabajo me permite vivir diversas experiencias & 1 & 2 & 3 & 4 & 5 \\
\hline 2 & Las cosas que aprendo en este trabajo hacen que lo valore más & 1 & 2 & 3 & 4 & 5 \\
\hline 3 & Este trabajo está en equilibrio con otras actividades de mi vida & 1 & 2 & 3 & 4 & 5 \\
\hline 4 & Este trabajo refleja las cualidades que valoro en mí & 1 & 2 & 3 & 4 & 5 \\
\hline 5 & Estoy completamente implicado con este trabajo & 1 & 2 & 3 & 4 & 5 \\
\hline 6 & Este trabajo me permite vivir experiencias inolvidables & 1 & 2 & 3 & 4 & 5 \\
\hline 7 & Este trabajo es una pasión que todavía logro controlar & 1 & 2 & 3 & 4 & 5 \\
\hline 8 & No puedo vivir sin este trabajo & 1 & 2 & 3 & 4 & 5 \\
\hline 9 & Dependo emocionalmente de este trabajo & 1 & 2 & 3 & 4 & 5 \\
\hline 10 & Me cuesta controlar la necesidad de realizar este trabajo & 1 & 2 & 3 & 4 & 5 \\
\hline 11 & El impulso es tan fuerte, que no puedo dejar de hacer este trabajo & 1 & 2 & 3 & 4 & 5 \\
\hline 12 & Tengo un sentimiento casi obsesivo por este trabajo & 1 & 2 & 3 & 4 & 5 \\
\hline 13 & Mi estado de ánimo depende de este trabajo & 1 & 2 & 3 & 4 & 5 \\
\hline 14 & No puedo concebir mi vida sin este trabajo & 1 & 2 & 3 & 4 & 5 \\
\hline
\end{tabular}

Fecha de recepción: Agosto de 2017.

Fecha de aceptación: Abril de 2019. 